\title{
Crossings and nestings in set partitions of classical types
}

\author{
Martin Rubey \\ Institut für Algebra, Zahlentheorie und Diskrete Mathematik \\ Leibniz Universität Hannover \\ martin.rubey@math.uni-hannover.de \\ http://wWw.iazd.uni-hannover.de/ rubey/ \\ Christian Stump \\ Centre de Recherches Mathmatiques \\ Université de Montréal \\ and Laboratoire de Combinatoire et d'Informatique Mathématique \\ Université du Québec à Montréal \\ christian.stump@univie.ac.at \\ http://homepage.univie.ac.at/christian.stump/
}

Submitted: Sep 8, 2009; Accepted: Jun 14, 2010; Published: Sep 1, 2010

Mathematics Subject Classifications: 05E15, 05A18

\begin{abstract}
In this article, we investigate bijections on various classes of set partitions of classical types that preserve openers and closers. On the one hand we present bijections for types $B$ and $C$ that interchange crossings and nestings, which generalize a construction by Kasraoui and Zeng for type $A$. On the other hand we generalize a bijection to type $B$ and $C$ that interchanges the cardinality of a maximal crossing with the cardinality of a maximal nesting, as given by Chen, Deng, Du, Stanley and Yan for type $A$.

For type $D$, we were only able to construct a bijection between non-crossing and non-nesting set partitions. For all classical types we show that the set of openers and the set of closers determine a non-crossing or non-nesting set partition essentially uniquely.
\end{abstract}




\section{Contents}

1 Set partitions for classical types $\quad 3$

2 Crossings and nestings in set partitions of type $A$

$\begin{array}{lll}3 & \text { Crossings and nestings in set partitions of type } C & 6\end{array}$

4 Crossings and nestings in set partitions of type $B \quad 8$

5 Non-crossing and non-nesting set partitions in type $D$

$6 \quad k$-crossing and $k$-nesting set partitions of type $C \quad 13$

\section{Introduction}

The lattice of non-crossing set partitions was first considered by Germain Kreweras in 15. It was later reinterpreted by Paul Edelman, Rodica Simion and Daniel Ullman, as a well-behaved sub-lattice of the intersection lattice for the hyperplane arrangement of type A, see e.g. [6, 17, 19]. Natural combinatorial interpretations of non-crossing partitions for the classical reflection groups were then given by Christos Athanasiadis and Vic Reiner in [3, 17].

On the other hand, non-nesting partitions were simultaneously introduced for all crystallographic reflection groups by Alex Postnikov as anti-chains in the associated root poset, see [17. Remark 2].

Within the last years, several bijections between non-crossing and non-nesting partitions have been constructed. In particular, type (i.e., block-size) preserving bijections were given by Christos Athanasiadis [2] for type $A$ and by Alex Fink and Benjamin Giraldo [8] for the other classical reflection groups. One of the authors of the present article [21] constructed another bijection for types $A$ and $B$ which transports other natural statistics. Recently, Ricardo Mamede and Alessandro Conflitti [5, 16] constructed bijections for types $A, B$ and $D$ which turn out to be subsumed by the bijections we present here.

The material on non-crossing partitions on the one hand and on non-nesting partitions on the other hand suggests that they are not only counted by the same numbers, namely the Catalan numbers, but are more deeply connected. These connections were presented by Drew Armstrong in [1, Chapter 5.1.3]. In this paper we would like to exhibit some further connections.

In the case of set partitions of type $A$, also the number of crossings and nestings was considered: Anisse Kasraoui and Jiang Zeng constructed a bijection which interchanges crossings and nestings in [13. Finally, in a rather different direction, William Chen, Eva Deng, Rosena Du, Richard Stanley and Catherine Yan [4] have shown that the number of set partitions where a maximal crossing has cardinality $k$ and a maximal nesting has cardinality $\ell$ is the same as the number of set partitions where a maximal crossing has cardinality $\ell$ and a maximal nesting has cardinality $k$. 


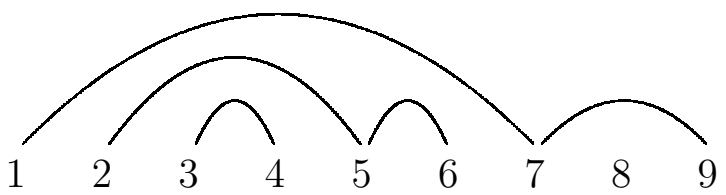

(a)

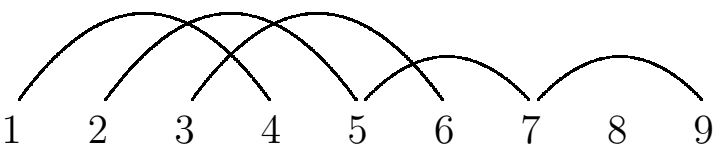

(b)

Figure 1: A non-crossing (a) and a non-nesting (b) set partition of [9].

In this paper, we present bijections on various classes of set partitions of classical types that preserve openers and closers. In particular, the bijection by Anisse Kasraoui and Jiang Zeng as well as the bijection by William Chen, Eva Deng, Rosena Du, Richard Stanley enjoy this property. We give generalizations of these bijections for the other classical reflection groups, whenever possible. Furthermore we show that the bijection is in fact (essentially) unique for the class of non-crossing and non-nesting set partitions.

\section{Set partitions for classical types}

A set partition of $[n]:=\{1,2,3, \ldots, n\}$ is a collection $\mathcal{B}$ of pairwise disjoint, non-empty subsets of $[n]$, called blocks, whose union is $[n]$. We visualize $\mathcal{B}$ by placing the numbers $1,2, \ldots, n$ in this order on a line and then joining consecutive elements of each block by an arc, see Figure 1 for examples.

The openers $\operatorname{op}(\mathcal{B})$ are the non-maximal elements of the blocks in $\mathcal{B}$, whereas the closers $\operatorname{cl}(\mathcal{B})$ are its non-minimal elements. For example, the set partitions in Figure 1 both have $\operatorname{op}(\mathcal{B})=\{1,2,3,5,7\}$ and $\operatorname{cl}(\mathcal{B})=\{4,5,6,7,9\}$.

A pair $(\mathcal{O}, \mathcal{C}) \subseteq[n] \times[n]$ is an opener-closer configuration, if $|\mathcal{O}|=|\mathcal{C}|$ and

$$
|\mathcal{O} \cap[k]| \geqslant|\mathcal{C} \cap[k+1]| \quad \text { for } \quad k \in\{0,1, \ldots, n-1\},
$$

or, equivalently, $(\mathcal{O}, \mathcal{C})=(\operatorname{op}(\mathcal{B}), \operatorname{cl}(\mathcal{B}))$ for some set partition $\mathcal{B}$ of $n$.

We remark that in [13], Anisse Kasraoui and Jiang Zeng distinguish between openers, closers and transients, which are, in our definition, those numbers which are both openers and closers.

It is now well established that set partitions of $[n]$ are in natural bijection with intersections of the reflecting hyperplanes $x_{i}-x_{j}=0$ in $\mathbb{R}^{n}$ of the Coxeter group of type $\mathrm{A}_{n-1}$. For example, the set partition in Figure 1(a) corresponds to the intersection

$$
\left\{x \in \mathbb{R}^{9}: x_{1}=x_{7}=x_{9}, x_{2}=x_{5}=x_{6}, x_{3}=x_{4}\right\} .
$$


Therefore, set partitions of $[n]$ can be seen as set partitions of type $\mathrm{A}_{n-1}$ and set partitions of other types can be defined by analogy, see [2, 17]. The reflecting hyperplanes for $B_{n}$ and $C_{n}$ are

$$
\begin{aligned}
& x_{i}=0 \text { for } 1 \leqslant i \leqslant n, \\
& x_{i}-x_{j}=0 \text { for } 1 \leqslant i<j \leqslant n, \text { and } \\
& x_{i}+x_{j}=0 \text { for } 1 \leqslant i<j \leqslant n .
\end{aligned}
$$

Thus, a set partition of type $B_{n}$ or $C_{n}$ is a set partition $\mathcal{B}$ of

$$
[ \pm n]:=\{1,2, \ldots, n,-1,-2, \ldots,-n\},
$$

such that

$$
B \in \mathcal{B} \Leftrightarrow-B \in \mathcal{B}
$$

and such that there exists at most one block $B_{0} \in \mathcal{B}$ (called the zero block) for which $B_{0}=-B_{0}$.

The hyperplanes for $D_{n}$ are those for $B_{n}$ and $C_{n}$ other than $x_{i}=0$ for $1 \leqslant i \leqslant n$, whence a set partition $\mathcal{B}$ of type $D_{n}$ is a set partition of type $B_{n}$ (or $C_{n}$ ) where the zero block, if present, must not consist of a single pair $\{i,-i\}$.

\section{Crossings and nestings in set partitions of type $A$}

One of the goals of this article is to refine the following well known correspondences between non-crossing and non-nesting set partitions. For ordinary set partitions, a crossing consists of a pair of $\operatorname{arcs}(i, j)$ and $\left(i^{\prime}, j^{\prime}\right)$ such that $i<i^{\prime}<j<j^{\prime}$,

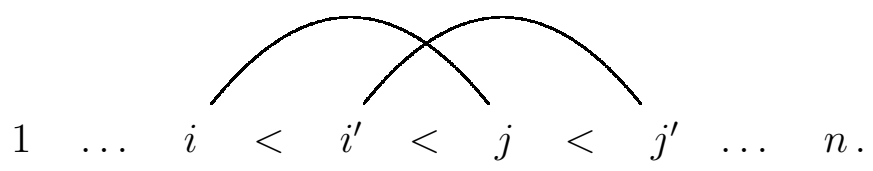

On the other hand, a nesting consists of a pair of $\operatorname{arcs}(i, j)$ and $\left(i^{\prime}, j^{\prime}\right)$ such that $i<i^{\prime}<$ $j^{\prime}<j$,

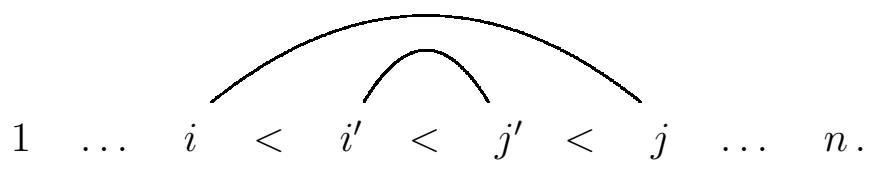

A set partition of $[n]$ is called non-crossing (resp. non-nesting) if the number of crossings (resp. the number of nestings) equals 0 .

It has been known for a long time that the numbers of non-crossing and non-nesting set-partitions of $[n]$ coincide. More recently, Anisse Kasraoui and Jiang Zeng have shown in [13] that much more is true: 
Theorem 2.1. There is an explicit bijection on set partitions of $[n]$, preserving the set of openers and the set of closers, and interchanging the number of crossings and the number of nestings.

The construction in [13] also proves the following corollary:

Corollary 2.2. For any opener-closer configuration $(\mathcal{O}, \mathcal{C}) \subseteq[n] \times[n]$, there exists a unique non-crossing set partition $\mathcal{B}$ of $[n]$ and a unique non-nesting set partition $\mathcal{B}^{\prime}$ of $[n]$ such that

$$
\operatorname{op}(\mathcal{B})=\operatorname{op}\left(\mathcal{B}^{\prime}\right)=\mathcal{O} \quad \text { and } \quad \operatorname{cl}(\mathcal{B})=\operatorname{cl}\left(\mathcal{B}^{\prime}\right)=\mathcal{C} .
$$

In the following section we will prove a statement for type $C$ completely analogous to the one of Anisse Kasraoui and Jiang Zeng.

Apart from the number of crossings or nestings, another natural statistic to consider is the cardinality of a 'maximal crossing' and of a 'maximal nesting': a maximal crossing of a set partition is a set of largest cardinality of mutually crossing arcs, whereas a maximal nesting is a set of largest cardinality of mutually nesting arcs. For example, in Figure 1(a), the arcs $\{(1,7),(2,5),(3,4)\}$ form a maximal nesting of cardinality 3. In Figure प(b) the arcs $\{(1,4),(2,5),(3,6)\}$ form a maximal crossing.

The following symmetry property was shown by William Chen, Eva Deng, Rosena Du, Richard Stanley and Catherine Yan [4]:

Theorem 2.3. There is an explicit bijection on set partitions, preserving the set of openers and the set of closers, and interchanging the cardinalities of a maximal crossing and a maximal nesting.

Since a 'maximal crossing' of a non-crossing partition and a 'maximal nesting' of a non-nesting partition both have cardinality 1, Corollary 2.2 implies that this bijection coincides with the bijection by Anisse Kasraoui and Jiang Zeng for non-crossing and nonnesting partitions. In particular, we obtain the curious fact that in this case, the bijection maps non-crossing partitions with $k$ nestings and maximal nesting having cardinality $\ell$ to non-nesting partitions with $k$ crossings and maximal crossing having cardinality $\ell$.

We have to stress however, that in general it is not possible to interchange the number of crossings and the cardinality of a maximal crossing with the number of nestings and the cardinality of a maximal nesting simultaneously.

Example 2.4. For $n=8$, there is a set partition with one crossing, six nestings and the cardinalities of a maximal crossing and a maximal nesting equal both one, namely $\{\{1,7\},\{2,8\},\{3,4,5,6\}\}$. However, there is no set partition with six crossings, one nesting and cardinalities of a maximal crossing and a maximal nesting equal to one. To check, the four set partitions with six crossings and one nesting are

$$
\begin{aligned}
& \{\{1,4,6\},\{2,5,8\},\{3,7\}\}, \\
& \{\{1,4,7\},\{3,5,8\},\{2,6\}\}, \\
& \{\{1,4,8\},\{2,5,7\},\{3,6\}\}, \\
& \{\{1,5,8\},\{2,4,7\},\{3,6\}\} .
\end{aligned}
$$




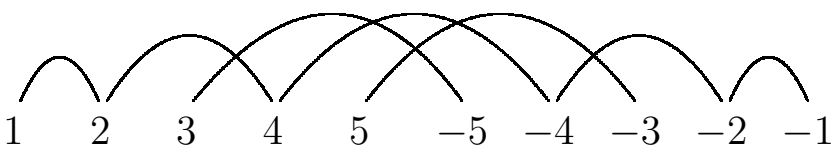

(a)

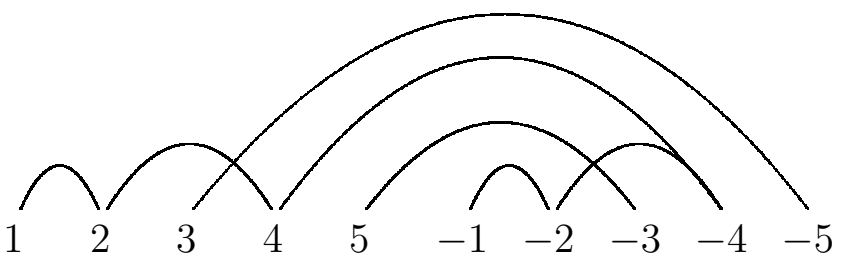

(b)

Figure 2: The nesting (a) and the crossing (b) diagram of a set partition of type $C_{5}$.

\section{Crossings and nestings in set partitions of type $C$}

Type independent definitions for non-crossing and non-nesting set partitions have been available for a while now, see for example [1, 2, 3, 17]. However, it turns out that the notions of crossing and nesting is more tricky, and we do not have a type independent definition. In this section we generalize the results of the previous section to type $C$.

We want to associate two pictures to each set partition, namely the 'crossing' and the 'nesting diagram'. To this end, we define two orderings on the set $[ \pm n]$ : the nesting order for type $C_{n}$ is

$$
1<2<\cdots<n<-n<\cdots<-2<-1,
$$

whereas the crossing order is

$$
1<2<\cdots<n<-1<-2<\cdots<-n .
$$

The nesting diagram of a set partition $\mathcal{B}$ of type $C_{n}$ is obtained by placing the numbers in $[ \pm n]$ in nesting order on a line and then joining consecutive elements of each block of $\mathcal{B}$ by an arc, see Figure 2(a) for an example.

The crossing diagram of a set partition $\mathcal{B}$ of type $C_{n}$ is obtained from the nesting diagram by reversing the order of the negative numbers. More precisely, we place the numbers in $[ \pm n]$ in crossing order on a line and then join consecutive elements in the nesting order of each block of $\mathcal{B}$ by an arc, see Figure 2(b) for an example. We stress that the same elements are joined by arcs in both diagrams. Observe furthermore that the symmetry property (11) implies that if $(i, j)$ is an arc, then its negative $(-j,-i)$ is also an arc.

A crossing is a pair of arcs that crosses in the crossing diagram, and a nesting is a pair of arcs that nests in the nesting diagram.

The openers $\operatorname{op}(\mathcal{B})$ are the positive non-maximal elements of the blocks in $\mathcal{B}$, the closers $\operatorname{cl}(\mathcal{B})$ the positive non-minimal elements. Thus, openers and closers are the start and end points of the arcs in the positive part of the nesting (or crossing) diagram. For example, the set partition displayed in Figure 2 has openers $\{1,2,3,4,5\}$ and closers $\{2,4\}$. For convenience, we call the negatives of the elements in $\operatorname{op}(\mathcal{B})$ negative closers and the negatives of the elements in $\operatorname{cl}(\mathcal{B})$ negative openers. 
In type $C_{n},(\mathcal{O}, \mathcal{C}) \subseteq[n] \times[n]$ is an opener-closer configuration, if

$$
|\mathcal{O} \cap[k]| \geqslant|\mathcal{C} \cap[k+1]| \quad \text { for } k \in\{0,1, \ldots, n-1\} .
$$

Note that we do not require that $|\mathcal{O}|=|\mathcal{C}|$.

Theorem 3.1. There is an explicit bijection on set partitions of type $C_{n}$, preserving the set of openers and the set of closers, and interchanging the number of crossings and the number of nestings.

Remark. In fact, the proof of this statement will show that we could also define crossing and nesting slightly differently. Namely, the statement of the theorem remains valid if we do not count crossings and nestings that involve an arc connecting two negative elements.

Furthermore, we will also see the following analog of Corollary 2.2

Corollary 3.2. For any opener-closer configuration $(\mathcal{O}, \mathcal{C}) \subseteq[n] \times[n]$, there exists a unique non-crossing set partition $\mathcal{B}$ and a unique non-nesting set partition $\mathcal{B}^{\prime}$, both of type $C_{n}$, such that

$$
\operatorname{op}(\mathcal{B})=\operatorname{op}\left(\mathcal{B}^{\prime}\right)=\mathcal{O} \quad \text { and } \quad \operatorname{cl}(\mathcal{B})=\operatorname{cl}\left(\mathcal{B}^{\prime}\right)=\mathcal{C} .
$$

Proof. The bijection proceeds in three steps. In the first step we consider only the given opener-closer configuration, and connect every closer, starting with the smallest, with the appropriate opener. Let us call an opener active, if it has not yet been connected with a closer.

Let $\mathcal{B}$ be a set partition of type $C_{n}$. Every closer $j \in \operatorname{cl}(\mathcal{B})$ (positive by definition) corresponds to an arc $(i, j)$ in the given set partition. This arc is nested by precisely those $\operatorname{arcs}\left(i^{\prime}, j^{\prime}\right)$ with $1 \leqslant i^{\prime}<i$ and either $j<j^{\prime} \leqslant n$ or $j^{\prime}$ negative. On the other hand, it is crossed by those arcs $\left(i^{\prime}, j^{\prime}\right)$ with $i<i^{\prime}<j$ and either $j<j^{\prime} \leqslant n$ or $j^{\prime}$ negative.

To construct the image of $\mathcal{B}$, we want to interchange the number of arcs crossing the arc $(i, j)$ with the number of arcs nesting it. Thus, if there are $k$ active openers smaller than $j$, and $(i, j)$ is crossed by $c$ arcs in $\mathcal{B}$, we connect $j$ with the $(c+1)^{\text {st }}$ active opener. Then, the arc $(i, j)$ will be nested by precisely $c$ arcs. The first step is completed when all closers in $\operatorname{cl}(\mathcal{B})$ have been connected.

Note that we do not have any choice if we want to construct, say, a non-nesting set partition: by connecting $j$ with any active opener except the first, we will produce a nesting.

In the second step, we use the symmetry property (1D) to connect elements $\left(i^{\prime}, j^{\prime}\right)$ with both $i^{\prime}$ and $j^{\prime}$ negative. More precisely, for every arc $(i, j)$ with $j \geqslant 1$, we add an arc $(-j,-i)$ to the set partition we are constructing.

Finally, we need to connect the remaining active openers with appropriate negative closers. Observe that two arcs $(i, j)$ and $\left(i^{\prime}, j^{\prime}\right)$ where both $i$ and $i^{\prime}$ are positive and both $j$ and $j^{\prime}$ are negative cross if and only if they nest. Suppose that the arcs connecting positive with negative elements in $\mathcal{B}$ are $\left\{\left(i_{1}, j_{1}\right),\left(i_{2}, j_{2}\right), \ldots,\left(i_{k}, j_{k}\right)\right\}$. Obviously, the set 
$\left\{i_{1}, i_{2}, \ldots, i_{k}\right\}$ and $\left\{-j_{1},-j_{2}, \ldots,-j_{k}\right\}$ are identical, and the arcs define a matching $\sigma$, such that $j_{m}=-i_{\sigma(m)}$.

Thus, if the remaining active openers are $\left\{o_{1}, o_{2}, \ldots, o_{k}\right\}$, the image of $\mathcal{B}$ shall contain the arcs $\left\{\left(o_{1},-o_{\sigma(1)}\right),\left(o_{2},-o_{\sigma(2)}\right), \ldots,\left(o_{k},-o_{\sigma(k)}\right)\right\}$. This completes the description of the bijection.

Again, note that we do not have any choice if we want to construct a non-nesting or non-crossing set partition: there is only one non-crossing - and therefore only one nonnesting - matching of the appropriate size that satisfies the symmetry property (II).

In Section 6 we will show the following analog to Theorem 2.3, where the definition of maximal crossing is as in type $A$ :

Theorem 3.3. There is an explicit bijection on set partitions of type $C_{n}$, preserving the set of openers and the set of closers, and interchanging the cardinalities of a maximal crossing and a maximal nesting.

Remark. It is tempting to consider a different notion of crossing and nesting, as suggested by Drew Armstrong in [1]. He defined a bump as an equivalence class of arcs, where the $\operatorname{arc}(i, j)$ is identified with $(-j,-i)$. From an algebraic point of view this is a very natural idea, since both correspond to the same hyperplane $x_{|i|}= \pm x_{|j|}$.

As an example, the partition $\{(1,4,-2),(3,5)\}$ would then be 3 -crossing, since with this definition $(1,4)$ crosses $(3,5)$ but also $(2,-4)=(4,-2)$. We were quite disappointed to discover that with this definition, all theorems in the present section would cease to hold.

\section{Crossings and nestings in set partitions of type $B$}

The definition of non-crossing set partitions of type $B_{n}$ coincides with the definition in type $C_{n}$, and the crossing diagram is also the same. However, the combinatorial model for non-nesting set partitions changes slightly: we define the nesting order for type $B_{n}$ as

$$
1<2<\cdots<n<0<-n<\cdots<-2<-1 \text {. }
$$

The nesting diagram of a set partition $\mathcal{B}$ is then obtained by placing the numbers in $[ \pm n] \cup 0$ in nesting order on a line and joining consecutive elements of each block of $\mathcal{B}$ by an arc, where the zero block is augmented by the number 0, if present. See Figure 3(a) for an example. The definition of openers $\operatorname{op}(\mathcal{B})$ and $\operatorname{closers} \operatorname{cl}(\mathcal{B})$ is the same as in type $C$, the number 0 is neither an opener nor a closer.

These changes are actually dictated by the general, type independent definitions for non-crossing and non-nesting set partitions. Moreover, it turns out that we need to ignore certain crossings and nestings that appear in the diagrams: a crossing is a pair of arcs that crosses in the crossing diagram, except if both arcs connect a positive and a negative element and at least one of them connects a positive element with an element smaller in absolute value. Pictorially, 


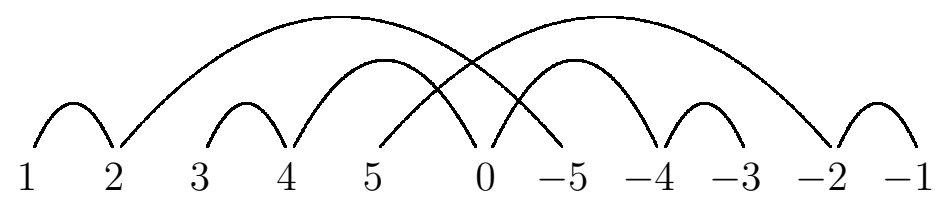

(a)

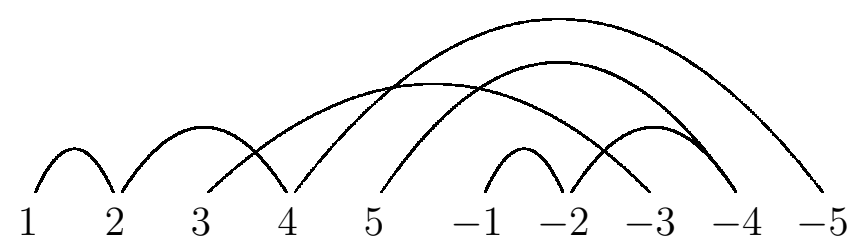

(b)

Figure 3: The nesting (a) and the crossing (b) diagram of a set partition of type $B_{5}$.

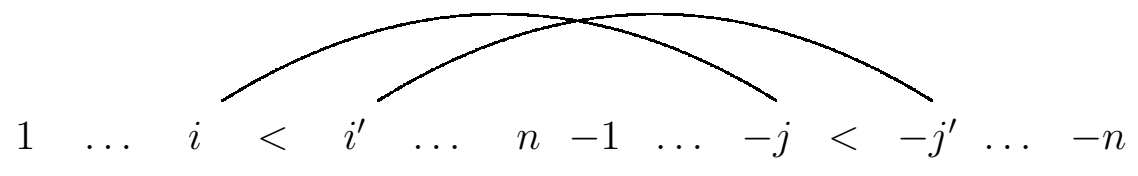

is not a crossing, if $j<i$ or $j^{\prime}<i^{\prime}$.

Similarly, a nesting is a pair of arcs that nests in the nesting diagram, except if both arcs connect a positive element or 0 with a negative element or 0 , and at least one of them connects a positive element with an element smaller in absolute value.

Example 4.1. The set partition in Figure 3 (b) has three crossings: $(3,-3)$ crosses $(2,4)$, $(4,-5)$, and $(-4,-2)$. It does not cross $(5,-4)$ by definition.

The set partition in Figure 3(a) has three nestings: $(2,-5)$ nests $(3,4)$ and $(4,0)$, and $(5,-2)$ nests $(-4,-3)$. However, $(5,-2)$ does not nest $(0,-4)$ by definition.

With this definition, we have a theorem that is only slightly weaker than in type $C$ :

Theorem 4.2. There is an explicit bijection on set partitions of type $B_{n}$, preserving the set of openers and the set of closers, and mapping the number of nestings to the number of crossings.

Again, we obtain an analog of Corollary 2.2.

Corollary 4.3. For any opener-closer configuration $(\mathcal{O}, \mathcal{C}) \subseteq[n] \times[n]$, there exists a unique non-crossing set partition $\mathcal{B}$ and a unique non-nesting set partition $\mathcal{B}^{\prime}$, both of type $B_{n}$, such that

$$
\operatorname{op}(\mathcal{B})=\operatorname{op}\left(\mathcal{B}^{\prime}\right)=\mathcal{O} \quad \text { and } \quad \operatorname{cl}(\mathcal{B})=\operatorname{cl}\left(\mathcal{B}^{\prime}\right)=\mathcal{C} .
$$

Proof. The first two steps of the bijection described in the proof of 3.1 can be reused unmodified for the present situation. However, it is no longer the case that the notions of nesting and crossing coincide for $\operatorname{arcs}(i, j)$ and $\left(i^{\prime}, j^{\prime}\right)$ with $i, i^{\prime} \geqslant 0$ and $j, j^{\prime} \leqslant 0$. 
We remark that there is still exactly one non-nesting way to connect the remaining active openers $\left\{o_{1}, o_{2}, \ldots, o_{k}\right\}$ with their negative counterparts, and the number 0 if $k$ is odd, such that the zero block contains 0 and the symmetry property (11) is satisfied. For

example, the situation for $k=3$ is as follows:

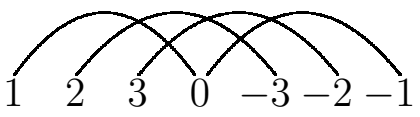

It remains to describe more generally a bijection that maps a type $B_{n}$ set partition $\mathcal{B}$ with opener-closer configuration $(\mathcal{O}, \mathcal{C})=([k], \emptyset)$ with $\ell$ nestings to a type $B_{n}$ set partition with $\ell$ crossings, and the same opener-closer configuration. In fact, we will really map $\mathcal{B}$ to a type $C_{n}$ set partition, such that there are exactly $\ell$ nestings occurring in the set of $\operatorname{arcs}(o, c)$ with $o<|c|$. This is sufficient, since for type $C_{n}$ set partitions, two $\operatorname{arcs}(i, j)$ and $\left(i^{\prime}, j^{\prime}\right)$ where both $i$ and $i^{\prime}$ are positive and both $j$ and $j^{\prime}$ are negative cross if and only if they nest.

If $\mathcal{B}$ does not contain a zero block, the image under the bijection is $\mathcal{B}$ itself. Otherwise, suppose that $\mathcal{B}$ consists of arcs

$$
\left(o_{1}, c_{1}=0\right),\left(o_{2}, c_{2}\right), \ldots,\left(o_{m}, c_{m}\right),
$$

with $o_{i} \leqslant\left|c_{i}\right|$ for $i>1$, together with their negatives. We assume furthermore that $\left|c_{2}\right|>\left|c_{3}\right|>\cdots>\left|c_{m}\right|$, i.e., the closers appear in nesting order.

Now let $j$ be minimal such that $o_{j}>\left|c_{j+1}\right|$, or, if no such $j$ exists, set $j:=m$. We then set

$$
\left(\tilde{o}_{i}, \tilde{c}_{i}\right):= \begin{cases}\left(o_{i}, c_{i+1}\right) & \text { for } i<j \\ \left(o_{i},-o_{i}\right) & \text { for } i=j \\ \left(o_{i}, c_{i}\right) & \text { for } i>j .\end{cases}
$$

We need to show that the number of nestings among

$$
\left(\tilde{o}_{1}, \tilde{c}_{1}\right),\left(\tilde{o}_{2}, \tilde{c}_{2}\right), \ldots,\left(\tilde{o}_{m}, \tilde{c}_{m}\right)
$$

is the same as in the original set of arcs. It is sufficient to show $\tilde{c}_{j-1}<\tilde{c}_{j}<\tilde{c}_{j+1}$, i.e., $c_{j}<-o_{j}<c_{j+1}$, since all other order relations remain unchanged. The relation $o_{j}<-c_{j}$ was required for all arcs, and $o_{j}>-c_{j+1}$ follows from the definition of $j$.

Together with Theorem 3.3 the bijection employed in the previous proof also shows the following theorem:

Theorem 4.4. There is an explicit bijection on set partitions of type $B_{n}$, preserving the set of openers and the set of closers, and interchanging the cardinalities of a maximal crossing and a maximal nesting.

\section{Non-crossing and non-nesting set partitions in type $D$}

In type $D$ we do not have any good notion of crossing or nesting, we can only speak properly about non-crossing and non-nesting set partitions. 

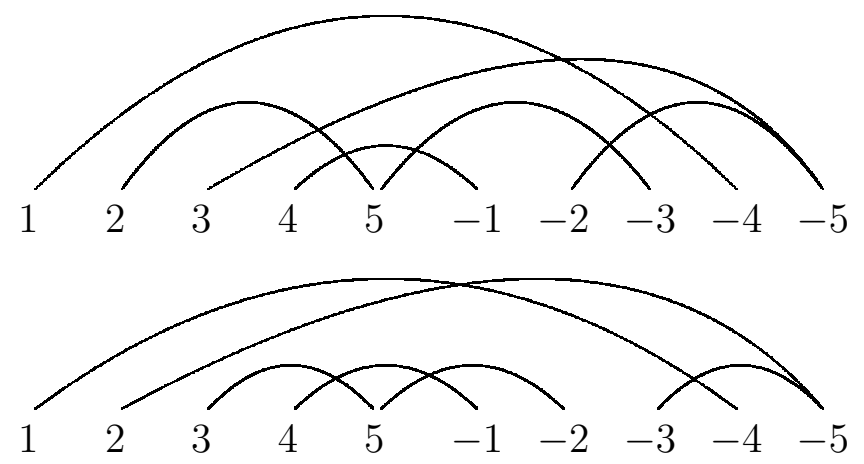

Figure 4: Two non-crossing set partition of type $D_{5}$. Both are obtained from each other by interchanging 5 and -5 .

A combinatorial model for non-crossing set partition of type $D_{n}$ was given by Christos Athanasiadis and Vic Reiner in [3]. For our purposes it is better to use a different description of the same model: let $\mathcal{B}$ be a set partition of type $D_{n}$ and let $\left\{\left(i_{1},-j_{1}\right), \ldots,\left(i_{k},-j_{k}\right)\right\}$ for positive $i_{\ell}, j_{\ell}<n$ be the ordered set of arcs in $\mathcal{B}$ starting in $\{1, \ldots, n-1\}$ and ending in its negative. $\mathcal{B}$ is called non-crossing if

(i) $(i,-i)$ is an $\operatorname{arc}$ in $\mathcal{B}$ implies $i=n$,

and if it is non-crossing in the sense of type $C_{n}$ with the following exceptions:

(ii) arcs in $\mathcal{B}$ containing $n$ must cross all arcs $\left(i_{\ell},-j_{\ell}\right)$ for $\ell>k / 2$,

(iii) $\operatorname{arcs}$ in $\mathcal{B}$ containing $-n$ must cross all $\operatorname{arcs}\left(i_{\ell},-j_{\ell}\right)$ for $\ell \leqslant k / 2$,

(iv) two arcs in $\mathcal{B}$ containing $n$ and $-n$ may cross.

Here, (i) is equivalent to say that if $\mathcal{B}$ contains a zero block $B_{0}$ then $n \in B_{0}$ and observe that (i) together with the non-crossing property of $\left\{\left(i_{1},-j_{1}\right), \ldots,\left(i_{k},-j_{k}\right)\right\}$ imply that $k / 2 \in \mathbb{N}$, see Figure 4 for an example.

Note that all conditions hold for a set partition $\mathcal{B}$ if and only if they hold for the set partition obtained from $\mathcal{B}$ by interchanging $n$ and $-n$.

A set partition of type $D_{n}$ is called non-nesting if it is non-nesting in the sense of [2]. This translates to our notation as follows: let $\mathcal{B}$ be a set partition of type $D_{n}$. Then $\mathcal{B}$ is called non-nesting if

(i) $(i,-i)$ is an $\operatorname{arc}$ in $\mathcal{B}$ implies $i=n$,

and if it is non-nesting in the sense of type $C_{n}$ with the following exceptions:

(ii) $\operatorname{arcs}(i,-n)$ and $(j, n)$ for positive $i<j<n$ in $\mathcal{B}$ are allowed to nest, as do

(iii) $\operatorname{arcs}(i,-j)$ and $(n,-n)$ for positive $k<i, j<n$ in $\mathcal{B}$ where $(k, n)$ is another arc in $\mathcal{B}$ (which exists by the definition of set partitions in type $D_{n}$ ). 

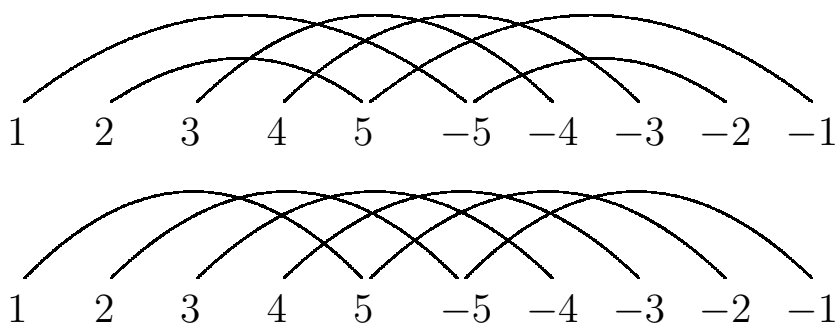

Figure 5: Two non-nesting set partition of type $D_{5}$. Both are obtained from each other by interchanging 5 and -5 .

Again, (i) means that if $B_{0} \in \mathcal{B}$ is a zero block then $n \in B_{0}$. (ii) and (iii) come from the fact that the positive roots $e_{i}+e_{n}$ and $e_{j}-e_{n}$ for $i \leqslant j$ are comparable in the root poset of type $C_{n}$ but are not comparable in the root poset of type $D_{n}$. As for non-crossing set partitions in type $D_{n}$, all conditions hold if and only if they hold for the set partition obtained by interchanging $n$ and $-n$. See Figure 5 for an example. The definition of openers $\operatorname{op}(\mathcal{B})$, closers $\operatorname{cl}(\mathcal{B})$ and opener-closer configuration is as in type $C$.

Proposition 5.1. Let $(\mathcal{O}, \mathcal{C}) \subseteq[n]$ be an opener-closer configuration. Then there exists a non-crossing set partition $\mathcal{B}$ of type $D_{n}$ with $\operatorname{op}(\mathcal{B})=\mathcal{O}$ and $\operatorname{cl}(\mathcal{B})=\mathcal{C}$ if and only if

$$
|\mathcal{O}|-|\mathcal{C}| \text { is even or } n \in \mathcal{O}, \mathcal{C} \text {. }
$$

Moreover, there exist exactly two non-crossing set partitions of type $D_{n}$ having this openercloser configuration if both conditions hold, otherwise, it is unique.

Proof. Suppose that $|\mathcal{O}|-|\mathcal{C}|$ is odd. Then the conditions to be non-crossing imply that we must have a zero block and therefore, $n$ must be an opener. On the other hand, the definition of set partitions of type $D_{n}$ implies that $n$ must be a closer. Thus, condition (2) is necessary. For the proof of the proposition we distinguish three cases:

Case 1: $|\mathcal{O}|=|\mathcal{C}|$. Then by the definition of opener-closer configurations, $n \notin O$ and the unique construction is the same as in the first step of the proof of Theorem 3.1 .

Case 2: $|\mathcal{O}|-|\mathcal{C}|$ is odd. Then by (2), $n$ is both opener and closer. For $\mathcal{C} \backslash\{n\}$ the construction is the same as in Case 1 . Now, there is an odd number of positive openers smaller than $n$ left. Connect the closer in $n$ to the unique opener in the middle as well as the opener in $-n$ to its negative. Connect $n$ and $-n$. Finally connect the remaining openers with there negative counterparts as closers such that they are non-crossing.

Case 3: $|\mathcal{O}|-|\mathcal{C}|>0$ is even. For $\mathcal{C} \backslash\{n\}$ the construction is again as in type $C_{n}$. Now, there is an even number of positive openers left. If $n$ is a closer but not an opener, then there is an odd number of positive openers smaller than $n$ left. Connect the closer in $n$ to the unique opener in the middle as well as the opener in $-n$ to its negative. If $n$ is a closer and also an opener then there is an even number of positive openers smaller than $n$ left. Connect the closer in $n$ to one of the two openers in the middle and the opener in $n$ to the negative of the other and also connect $-n$ to their negatives. This 
gives the two possibilities in this case and observe that both are obtained from each other by interchanging $n$ and $-n$. Finally connect the remaining openers with there negative counterparts as closers such that they are non-crossing.

As in types $A, B$ and $C$, the analogue proposition holds also for non-nesting set partitions of type $D_{n}$ :

Proposition 5.2. Let $(\mathcal{O}, \mathcal{C}) \subseteq[n]$ be an opener-closer configuration. Then there exists a non-nesting set partition $\mathcal{B}$ of type $D_{n}$ with $\mathrm{op}(\mathcal{B})=\mathcal{O}$ and $\mathrm{cl}(\mathcal{B})=\mathcal{C}$ if and only if

$$
|\mathcal{O}|-|\mathcal{C}| \text { is even or } n \in \mathcal{O}, \mathcal{C} \text {. }
$$

Furthermore, there exist exactly two non-nesting set partitions of type $D_{n}$ having this opener-closer configuration if both conditions hold, otherwise, it is unique.

Proof. The proof that condition (31) is necessary is analogous to the proof in the noncrossing case.

Recall that a set partition of type $D_{n}$ is non-nesting if it is non-nesting in the sense of type $C_{n}$ except for arcs of the forms

(i) $\operatorname{arcs}(i,-n)$ and $(j, n)$ for positive $i<j<n$,

(ii) $\operatorname{arcs}(i,-j)$ and $(n,-n)$ for positive $k<i, j<n$ where $(k, n)$ is another arc (which exists if $(n,-n)$ is an arc),

and observe that in both cases, $n$ is both an opener and a closer. Therefore, the construction is exactly the same as in type $C_{n}$ otherwise. We now prove the remaining two cases:

Case 1: $|\mathcal{O}|-|\mathcal{C}|$ is odd. The unique possibility is to connect $n$ and $-n$ and all others in the same way as in type $C_{n}$. All nesting arcs in this case are of the form (ii).

Case 2: $|\mathcal{O}|-|\mathcal{C}|$ is even. In this case, we have two possibilities: the first is to connect closers and openers as in type $C_{n}$ without creating any nestings. The second is to connect the closers in $\mathcal{C} \backslash\{n\}$ as above to the associated openers, then we connect $-n$ to the first active opener and $n$ to the associated negative closer. The remaining positive openers and their associated negative closers are finally connected such that they are non-nesting. All nesting arcs in this case are of the form (i). Observe also that possibilities 1 and 2 are obtained from each other by interchanging $n$ and $-n$.

\section{$6 k$-crossing and $k$-nesting set partitions of type $C$}

In this section we prove Theorem 3.3, which states that the cardinalities of a maximal crossing and a maximal nesting of type $C$ set partitions are equidistributed.

The rough idea of our bijection is as follows: we first show how to render a type $C_{n}$ set partition in the language of 0-1-fillings of a certain polyomino, as depicted in Figure 6(a). We will do this in such a way that maximal nestings correspond to north-east chains of ones of maximal length. 
Interpreting this filling as a growth diagram in the sense of Sergey Fomin and Tom Roby [9, 10, 11, 18, enables us to define a transformation on the filling that maps technicalities aside - the length of the longest north-east chain to the length of the longest south-east chain. This filling can then again be interpreted as a $C_{n}$ set partition, where south-east chains of maximal length correspond to maximal crossings. Many variants of the transformation involved are described in Christian Krattenthaler's article [14], we will employ yet another (slight) variation.

Let us now give a detailed description of the objects involved: a polyomino is a finite subset of $\mathbb{Z}^{2}$, where we regard an element of $\mathbb{Z}^{2}$ as a cell. A column of a polyomino is the set of cells along a vertical line, a row is the set of cells along a horizontal line. A trapezoid polyomino of size $n$ is the polyomino consisting of $n$ columns of height $2 n-1,2 n-2, \ldots, n$, arranged in this order. (We warn the reader that in other contexts, the name 'trapezoid polyomino' is used for more general polyominoes.)

A partial 0-1 filling of a polyomino is an assignment of 0's and 1's to its cells such that there is at most one 1 in each row and in each column. A north-east chain of length $k$ is a sequence of $k$ cells with entry 1 in a filling of a nesting polyomino, such that every cell is strictly to the right and strictly above the preceding cell in the sequence. Similarly, a south-east chain of length $k$ is a sequence of $k$ cells with entry 1 in a filling of a crossing polyomino, such that every cell is strictly to the right and strictly below the preceding cell in the sequence. Furthermore, we require that the smallest rectangle containing all cells of the sequence is completely contained in the polyomino. We remark that this condition is trivially satisfied for north-east chains.

The nesting polyomino for type $C_{n}$ set partitions is the trapezoid polyomino of size $n$, with columns labelled $1,2, \ldots, n$ and rows from top to bottom $2,3, \ldots, n,-n, \ldots,-2,-1$, as in Figure 6(a). Now, every box of the polyomino corresponds to an arc that may be present in a nesting diagram: an arc $(i, j)$ corresponds to the cell in column $i$, row $j$.

We encode a type $C_{n}$ set partition by placing ones into those boxes that correspond to arcs, and zeroes into the other boxes, as in Figure 6(a). For convenience, zeros are not shown and ones are indicated by crosses. (We ignore the integer partitions labelling the top-right corners for the moment.) A partial 0-1-filling of the nesting polyomino corresponds to a type $C_{n}$ set-partition if and only if

1. the restriction of the filling to the rows $-1,-2, \ldots,-n$ is symmetric with respect to the north-east diagonal as indicated in Figure 6(a), and

2. there is at most one non-zero entry on this diagonal.

The crossing polyomino for type $C_{n}$ set partitions is a polyomino of the same shape as the nesting polyomino. We label the columns $1,2, \ldots n$ as before. However, we now label the rows from top to bottom $2,3, \ldots, n,-1,-2, \ldots,-n$, as in Figure 6 (b). We find that a partial 0-1-filling of the crossing polyomino corresponds to a type $C_{n}$ set-partition under the same conditions as before, with the difference that the symmetry axis (indicated in the figure by a dotted line) now runs south-east instead of north-east. 

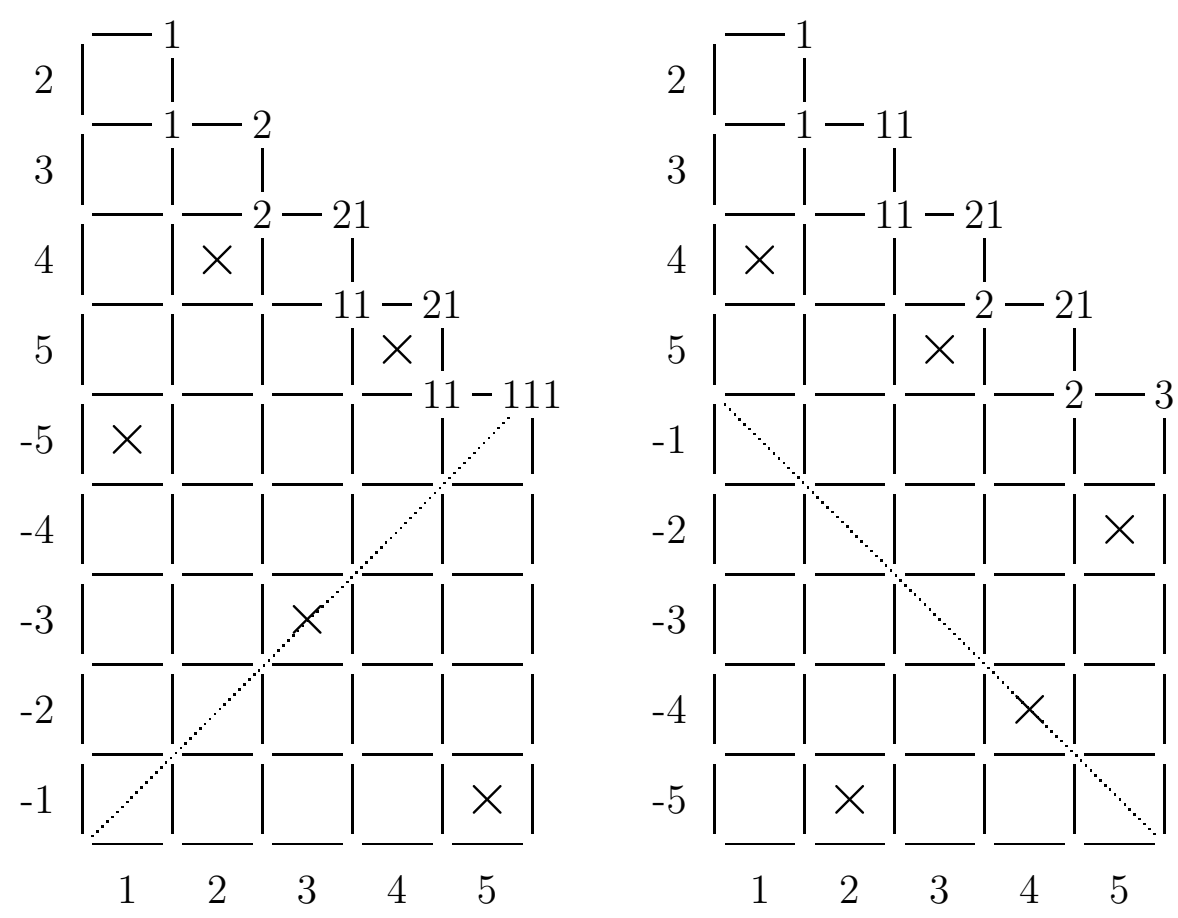

(a) $\{(1,-5,-4,-2),(3,-3)\}$ (b) $\{(1,4,-1,-4),(2,-5)\}$

Figure 6: The nesting polyomino (a) of a type $C_{5}$ set partition and the crossing polyomino (b) of its image, see proof of Theorem 3.3

Lemma 6.1. A longest north-east chain in a partial 0-1-filling of the nesting polyomino corresponds to a maximal nesting in the associated type $C_{n}$ set partition. Similarly, a longest south-east chain in a partial 0-1-filling of the crossing polyomino corresponds to a maximal crossing in the associated type $C_{n}$ set-partition.

Proof. The statement for the nesting polyomino is trivial: two arcs nest precisely when one of the corresponding crosses in the nesting polyomino is north-east of the other. For the crossing polyomino we have to show that for any maximal crossing involving arcs connecting two negative elements, there is another maximal crossing that does not involve such arcs.

We first note that a maximal crossing cannot contain an arc connecting two positive elements and an arc connecting two negative elements simultaneously, since these would not cross.

Thus, if a maximal crossing $\left(o_{1}, c_{1}\right),\left(o_{2}, c_{2}\right), \ldots,\left(o_{k}, c_{k}\right)$ involves an arc connecting two negative elements, all of $c_{1}, c_{2}, \ldots, c_{k}$ must be negative. By symmetry, the set of $\operatorname{arcs}\left(-c_{1},-o_{1}\right),\left(-c_{2},-o_{2}\right), \ldots,\left(-c_{k},-o_{k}\right)$ is also a maximal crossing containing no arc connecting two negative elements.

In the following we want to associate certain sequences of integer partitions to fillings of the nesting and the crossing polyomino that correspond to type $C_{n}$ set-partitions. 
A growth diagram is a labelling of the corners of the cells of a partial 0-1 filling of a polyomino with integer partitions according to the following rule: for any $\ell$, the sum of the first $\ell$ parts of each of these integer partitions is just the maximal cardinality of a union of $\ell$ north-east chains in the rectangular region of the polyomino to the left and below the corner. In particular, the first part of every partition gives the length of the longest north-east chain in this region.

The following proposition is a summary of the properties of growth diagram we need:

Proposition 6.2. Partial 0-1 fillings of a trapezoid polyomino of size $n$ are in bijection with sequences of integer partitions $\left(\lambda_{0}=\emptyset, \lambda_{1}, \ldots, \lambda_{3 n-1}=\emptyset\right)$, such that for $1 \leqslant k \leqslant n-1$

- $\lambda_{2 k-1}=\lambda_{2 k}$, or $\lambda_{2 k-1}$ is obtained from $\lambda_{2 k}$ by adding one to some part, and

- $\lambda_{2 k+1}=\lambda_{2 k}$, or $\lambda_{2 k+1}$ is obtained from $\lambda_{2 k}$ by adding one to some part,

and, for $2 n \leqslant k \leqslant 3 n-1, \lambda_{k-1}=\lambda_{k}$ or $\lambda_{k-1}$ is obtained from $\lambda_{k}$ by adding one to some part.

This sequence of partitions can be found by reading off the labels of the growth diagram along the upper-right border from top to bottom.

The inverse map can be described by so-called 'local rules': the integer partitions labelling all but the bottom-left corner of a cell determine the remaining integer partition and the content - 0 or 1 - of the cell.

Moreover, for every partition labelling a corner in the growth diagram, and for any $\ell$, the sum of the first $\ell$ parts of the conjugate (also referred to as: transposed) partition is just the maximal cardinality of a union of $\ell$ south-east chains in the rectangular region of the polyomino to the left and below the corner. In particular, the first part of every conjugated partition gives the length of the longest south-east chain in this region.

Proof. A detailed exposition, although without proofs can be found in Section 2 of Christian Krattenthaler's article [14. Proofs can be found (apart from Sergey Fomin's and Curtis Greene's original papers [11, 12] on the subject) in Section 7.13 and Section A1.1 of [20].

To be able to deal with the symmetries in the nesting and the crossing polyominoes, we need another two well-known facts. One of them involves an involution on sequences of integer partitions called evacuation, defined for example in A1.2.8 of [20]. It is not necessary to define it here, for our purposes it is indeed enough to know that evacuation is an involution.

Proposition 6.3. A partial 0-1 filling of a square polyomino is symmetric with respect to its north-east diagonal if and only if the sequence of integer partitions labelling its right border (read from bottom to top) is the same as the sequence of integer partitions labelling its upper border (read from left to right).

Moreover, the number of entries on the diagonal equal to 1 is given by the number of odd parts of the partition conjugate to the one labelling the top-right corner of the polyomino. 
A partial 0-1 filling of a square polyomino is symmetric with respect to its south-east diagonal if and only if the sequence of integer partitions labelling its right border (read from bottom to top) is obtained by evacuating the sequence of integer partitions labelling its upper border (read from left to right).

Moreover, the number of entries on the diagonal equal to 1 is given by the number of odd parts of the partition labelling the top-right corner of the polyomino.

Proof. The first statement is Corollary 7.13.6 in [20]. The interpretation of the number of 1's on the diagonal is Exercise 7.28a in the same reference.

The effect on the partitions of reflecting the filling on a vertical axis is also described in this reference, as Corollary A1.2.11. Clearly, a filling of a square polyomino is symmetric with respect to its south-east diagonal, if and only if the reflected filling is symmetric with respect to the north-east diagonal.

It is now easy to construct the desired bijection demonstrating Theorem 3.3.

Proof of Theorem 3.3. We first show that partial 0-1 fillings of nesting polyominoes corresponding to type $C_{n}$ set partitions are in bijection with sequences of integer partitions $\left(\lambda_{0}=\emptyset, \lambda_{1}, \ldots, \lambda_{2 n-1}\right)$ such that for $1 \leqslant k \leqslant n-1$

- $\lambda_{2 k-1}=\lambda_{2 k}$, or $\lambda_{2 k-1}$ is obtained from $\lambda_{2 k}$ by adding one to some part, and

- $\lambda_{2 k+1}=\lambda_{2 k}$, or $\lambda_{2 k+1}$ is obtained from $\lambda_{2 k}$ by adding one to some part,

and where the partition conjugate to $\lambda_{2 n-1}$ has at most one odd part. These sequences are given by the labels of the growth diagram along the upper-right border, up to and including the top-right corner of column $n$, row $-n$.

We now indicate how to recover the filling given only this sequence of partitions: using the 'local rules' mentioned in Proposition 6.2, we can recover the entries in rows 2 to $n$ of the 0-1-filling, as well as a sequence of integer partitions labelling the top-right corners of row $-n$. Since the filling of the square polyomino below row $-n$ should be symmetric, and there should be at most one entry 1 on the diagonal, Proposition 6.3 applies.

Very similarly, we can show that partial 0-1 fillings of crossing polyominoes corresponding to type $C_{n}$ set partitions are in bijection with sequences of integer partitions as above, except that now the partition $\lambda_{2 n-1}$ itself has at most one odd part.

Thus, to map a type $C_{n}$ set partition with a maximal nesting having cardinality $k$ to a set partition with maximal crossing having the same cardinality, we proceed as follows: first we compute the sequence of integer partitions $\left(\lambda_{0}=\emptyset, \lambda_{1}, \ldots, \lambda_{2 n-1}\right)$ as above, and then the filling of the crossing polyomino corresponding to $\left(\left(\lambda_{0}\right)^{t}=\emptyset,\left(\lambda_{1}\right)^{t}, \ldots,\left(\lambda_{2 n-1}\right)^{t}\right)$, where $\left(\lambda_{i}\right)^{t}$ denotes the partition conjugate to $\lambda_{i}$.

We have to remark that the bijection presented above is not an involution. Furthermore, it does not exchange the crossing and the nesting numbers. As a small example, consider the $C_{4}$ partition $\{1,4\},\{2,-3\}$, which is non-nesting, has four crossings, and the cardinality of a maximal crossing is two. Its crossing polyomino is mapped to the nesting polyomino of the $C_{4}$ partition $\{1,-3\},\{2,4\}$, which has two nestings, two crossings. Of course, by construction of the bijection, the cardinality of a maximal nesting is two, also. 


\section{References}

[1] Drew Armstrong, Generalized Noncrossing Partitions and Combinatorics of Coxeter Groups, Ph.D. thesis, Cornell University, 2007, math.CO/0611106v1, to appear in Mem. Amer. Math. Soc.

[2] Christos Athanasiadis, On noncrossing and nonnesting partitions for classical reflection groups, Electronic Journal of Combinatorics 5 (1998), Research Paper 42, 16 pp. (electronic). MR MR1644234 (99i:05204)

[3] Christos Athanasiadis and Victor Reiner, Noncrossing partitions for the group $D_{n}$, SIAM Journal on Discrete Mathematics 18 (2004), no. 2, 397-417 (electronic). MR MR2112514 (2006b:06004)

[4] William Chen, Eva Deng, Rosena Du, Richard Stanley, and Catherine Yan, Crossings and nestings of matchings and partitions, Transactions of the American Mathematical Society 359 (2007), no. 4, 1555-1575 (electronic), math.CO/0501230. MR MR2272140 (2007i:05015)

[5] Alessandro Conflitti and Ricardo Mamede, On noncrossing and nonnesting partitions of type D, Preprint (2009), math.CO/08905.4371.

[6] Paul Edelman, Chain enumeration and noncrossing partitions, Discrete Mathematics 31 (1980), no. 2, 171-180. MR MR583216 (81i:05018)

[7] Paul Edelman and Rodica Simion, Chains in the lattice of noncrossing partitions, Discrete Mathematics 126 (1994), no. 1-3, 107-119. MR MR1264480 (95f:05012)

[8] Alex Fink and Benjamin Iriarte Giraldo, Bijections between noncrossing and nonnesting partitions for classical reflection groups, Proceedings of the 21st International Conference on Formal Power Series and Algebraic Combinatorics, Discrete Mathematics and Theoretical Computer Science, DMTCS, 2009, math.CO/0810.2613v3, pp. 399-412.

[9] Sergey Fomin, The generalized Robinson-Schensted-Knuth correspondence, Zapiski Nauchnykh Seminarov Leningradskogo Otdeleniya Matematicheskogo Instituta imeni V. A. Steklova Akademii Nauk SSSR (LOMI) 155 (1986), no. Differentsialnaya Geometriya, Gruppy Li i Mekh. VIII, 156-175, 195. MR MR869582 (88b:06003)

[10] Sergey Fomin, Duality of graded graphs, Journal of Algebraic Combinatorics 3 (1994), no. 4, 357-404. MR MR1293822 (95i:05088)

[11] _ Schensted algorithms for dual graded graphs, Journal of Algebraic Combinatorics 4 (1995), no. 1, 5-45. MR MR1314558 (95m:05246)

[12] Curtis Greene, An extension of Schensted's theorem, Advances in Mathematics 14 (1974), 254-265. MR MR0354395 (50 \#6874)

[13] Anisse Kasraoui and Jiang Zeng, Distribution of crossings, nestings and alignments of two edges in matchings and partitions, Electronic Journal of Combinatorics 13 (2006), no. 1, Research Paper 33, 12 pp. (electronic), math.CO/0601081. MR MR2212506 (2006k:05021) 
[14] Christian Krattenthaler, Growth diagrams, and increasing and decreasing chains in fillings of Ferrers shapes, Advances in Applied Mathematics 37 (2006), no. 3, 404431, math.CO/0510676. MR MR2261181 (2007h:05011)

[15] Germain Kreweras, Sur les partitions non croisées d'un cycle, Discrete Mathematics 1 (1972), no. 4, 333-350. MR MR0309747 (46 \#8852)

[16] Ricardo Mamede, A bijection between noncrossing and nonnesting partitions of types $A$ and $B$, Proceedings of the 21st International Conference on Formal Power Series and Algebraic Combinatorics, Discrete Mathematics and Theoretical Computer Science, DMTCS, 2009, math.CO/0810.1422, pp. 599-612.

[17] Victor Reiner, Non-crossing partitions for classical reflection groups, Discrete Mathematics 177 (1997), no. 1-3, 195-222. MR MR1483446 (99f:06005)

[18] Tom Roby, Applications and extensions of Fomin's generalization of the RobinsonSchensted correspondence to differential posets, Ph.D. thesis, M.I.T., Cambridge, Massachusetts, 1991.

[19] Rodica Simion and Daniel Ullman, On the structure of the lattice of noncrossing partitions, Discrete Mathematics 98 (1991), no. 3, 193-206. MR MR1144402 (92j:06003)

[20] Richard Stanley, Enumerative combinatorics. Vol. 2, Cambridge Studies in Advanced Mathematics, vol. 62, Cambridge University Press, Cambridge, 1999, With a foreword by Gian-Carlo Rota and appendix 1 by Sergey Fomin. MR MR1676282 (2000k:05026)

[21] Christian Stump, Non-crossing partitions, non-nesting partitions and Coxeter sortable elements in types $A$ and B, Preprint (2008), math.CO/0808.2822. 